\title{
Defensins: ancient but potentially dangerous weapons in the armoury
}

\author{
David Burnett
}

The aphorism of Ecclesiastes that "there is no new thing under the sun" seems all too often to be irritatingly true; who has not had a "novel" idea that turned out to be "old hat"? The full quotation, read in context, seems to divine or explain the homologous and analogous forms found in the natural world, particularly now that structures are analysed at the molecular level. The defensins illustrate the point.

Defensins are microbicidal single chain peptides with a molecular weight of about 4 kilodaltons. They have been identified in the phagocytic leucocytes of several mammalian species and sequenced-four from man, six from the rabbit, four from the rat, and one from the guinea pig. ${ }^{1}$ All are rich in arginine residues and each molecule has six conserved cysteine residues and three disulphide bonds. Of the human defensins, found in the neutrophil azurophil granules, ${ }^{2}$ three (designated HNP 1-3) differ only by the last $N$-terminal amino acid $^{3}$; HNP-1 and HNP-3 are each 30 residues long and HNP-2 29. The fourth, HNP-4, is 33 residues long, has less structural homology with the other three, and is less abundant than them. ${ }^{34}$ The conserved nature of the mammalian defensins is illustrated by the similarities between human and rabbit defensins, which have 11 amino acid residues in common. ${ }^{4}$ More remarkable, however, was the discovery of bacteriocidal peptides, similar to defensins, in insects. Two peptides isolated from the larva of the fly Phormia terranovae are each 40 amino acids long, with the familiar six cysteines, and contain a 21 residue sequence in which 10 amino acids are identical to those of the rabbit defensin NP- $1^{5}$ and six to those of the human defensins HNP 1-3. Whether these are truly homologous peptides or examples of Mother Nature reinventing the proverbial wheel (defensins, incidentally, are cyclic molecules ${ }^{6}$ ) is not known and could be established only by tracing the evolution of the defensin gene throughout the animal kindom. Nevertheless, the presence of such a similar basic plan over what, phylogenetically, represents 300 million years suggests that it is an efficient design for microbial killing.

Defensins kill various bacteria, fungi, and enveloped viruses. ${ }^{2-9}$ The mechanism of killing depends on the formation of ion channels in the membranes of the target microbes. ${ }^{9}$
Why might defensins be dangerous? Because they are not selective in the membranes they are targeted at, they can also kill human cells, including the cells of various tumour cell lines and endothelial cells. ${ }^{10}$ They therefore have the potential to contribute to host cell injury. Recent investigation of the mechanism of cytotoxicity $^{11}$ produced some interesting results that provoke many questions. In contrast to microbial killing, defensins affect mammalian cells in two phases. Firstly, within five minutes there was evidence that small molecules were leaking out of and into the cells, suggesting the creation of small channels in the cell membrane; this phase lasted about 30 minutes. If the defensins were washed away at this stage the cell membranes recovered their integrity. If the defensins remained the cells became permeable to larger molecules (that is, lysed) after about one hour and this effect increased over six hours. This might represent a continuous process, with the holes in the cell membrane becoming progressively larger, but the two phases showed different sensitivities to added factors. For instance, whereas the second ("lytic") phase was enhanced by actinomycin D and cycloheximide (inhibitors of protein synthesis), the initial phase was not. These and other examples led to the conclusion that there are two distinct processes in the killing of mammalian cells by defensins. It was suggested that the lytic phase resulted from the entry of defensin molecules into the target cell. The defensins therefore have a potential for damaging host tissues, but do they?

To lyse a target cell the defensin must be expressed outside the azurophil granule-that is, effectively outside the neutrophil. Neutrophils can be induced, with high concentrations of phorbol ester or during experimental phagocytosis, to release small amounts of defensins $(8 \%$ and $3 \%$ respectively of their total). ${ }^{12}$ This and other studies ${ }^{13}$ show that neutrophils are not easily persuaded to "degranulate" their azurophil granules, which is fortunate in view of their contents. Nevertheless, the azurophil enzymes are found in pus and sputum, demonstrating that azurophil components are sometimes in an extracellular environment where they might cause damage to surrounding tissues. It has yet to be established whether defensins are also present and 
active in these secretions. In view of their potential danger, it might seem predictable that inhibitors of defensins should exist. Inhibition of defensin activity by serum has been described and was attributed to binding by albumin ${ }^{11}$ but has subsequently been shown to be due to $\alpha_{2}$ macroglobulin. ${ }^{14}$

Perhaps any extracellular defensin activity, be it cytotoxic or microbicidal, is confined largely to the pericellular space beneath adherent neutrophils; azurophil granules have been shown to be active in this environment, from which large inhibitors are excluded. ${ }^{1315}$ Nevertheless, the relatively long period required for cell lysis to be accomplished by defensins ${ }^{11}$ raises the question of whether neutrophils would remain on an individual target cell long enough for irreparable damage to occur. As defensins have been shown to act synergistically with hydrogen peroxide in causing cytolysis ${ }^{16}$ possibly the process is accelerated when the defensins are associated with metabolising neutrophils. So far, only purified defensins have been studied; any extracellular role of these molecules will be revealed by methods that can detect their presence and activity in and around neutrophils. Furthermore, it will be interesting to discover exactly how defensins create pores in biological membranes. Lichtenstein ${ }^{11}$ has speculated briefly on the possible mechanism. It may be identical to that of other lytic molecules, such as complement. On the other hand, it may be a distinct and unique process-but again perhaps there is no new thing under the sun.

1 Ganz T, Selsted ME, Lehrer RI. Defensins. Eur J Haematol 1990;44:1-8.
2 Ganz T, Selsted ME, Szklarek D, Harwig SSL, Daher KA, Bainton DF, et al. Defensins. Natural peptide antibiotics of human neutrophils. J Clin Invest 1985;76:1427-35.

3 Selsted ME, Harwig SSL, Ganz T, Schilling JW, Lehrer RI. Primary structure of three human neutrophil defensins. J Clin Invest 1985;76:1436-9.

4 Wilde GC, Griffith JE, Marra MN, Snable JL, Scott RW Purification and characterization of human neutrophil peptide 4, a new member of the defensin family. $J$ Biol Chem 1989;264:11200-3.

5 Lambert J, Keppi E, Dimarcq J-L, Wicker C, Reichhart $\mathrm{J}-\mathrm{M}$, Dunbar B, et al. Insect immunity: isolation from immune blood of the dipteran Phormia terranovae of two insect antibacterial peptides with sequence homology to insect antibacterial peptides with sequence homology to Acad Sci USA 1989;86:262-6.

6 Selsted ME, Harwig SS. Determination of the disulphide array in the human defensin HNP-2. A covalently cyclized peptide. J Biol Chem 1989;264:4003-7.

7 Daher KA, Selsted ME, Lehrer RI. Direct inactivation of viruses by human granulocyte defensins. J Virol $\vec{\circ}$ 1985;54:1068-74

8 Greenwald GI, Ganz T. Defensins mediate the microbicidal $\overrightarrow{\vec{\omega}}$ activity of human neutrophil granule extract against $\omega$ Acinetobacter calcoaceticus. Infect Immun 1987;55:1365-8.

9 Lehrer RI, Barton A, Daher KA, Harwig SS, Ganz T, Selsted ME. Interaction of human defensins with Escherichia coli: mechanism of bactericidal activity. J Clin Invest 1989;84:553-61.

10 Okrent DG, Lichtenstein AK, Ganz T. Direct cytotoxicity of polymorphonuclear leukocyte granule proteins to $f$ human lung-derived cells and endothelial cells. $\mathrm{Am} \mathrm{Rev}$ Respir Dis 1990;141:179-85.

11 Lichtenstein A. Mechanism of mammalian cell lysis mediated by peptide defensins. Evidence for an initial $>$ alteration of the plasma membrane. J Clin Invest 1991;88:93-100

12 Ganz T. Extracellular release of antimicrobial defensins by human polymorphonuclear leukocytes. Infect Immun 1987;55:568-71.

13 Chamba A, Afford SC, Stockley RA, Burnett D. Extracellular proteolysis of fibronectin by neutrophils: characterization and the effects of recombinant cytokines. Am J Respir Cell Molec Biol 1991;4:330-7.

14 Panyutich A, Ganz T. Activated $\alpha_{2}$-macroglobulin is a principal defensin-binding protein. Am J Respir Cell Molec Biol 1991;5:101-6.

15 Campbell EJ, Campbell MA. Pericellular proteolysis by neutrophils in the presence of proteinase inhibitors: effects of substrate opsonization. J Cell Biol 1988;106:667-76.

16 Lichtenstein A, Ganz T, Selsted ME, Lehrer RI. Synergistic cytolysis mediated by hydrogen peroxide combined with peptide defensins. Cell Immunol 1988;114:104-10. 